\title{
Updating Street Maps using Changes Detected in Satellite Imagery
}

\author{
Favyen Bastani ${ }^{1}$, Songtao $\mathrm{He}^{1}$, Satvat Jagwani ${ }^{1}$, Mohammad Alizadeh ${ }^{1}$, \\ Hari Balakrishnan ${ }^{1}$, Sanjay Chawla ${ }^{2}$, Sam Madden ${ }^{1}$, Mohammad Amin Sadeghi ${ }^{2}$ \\ ${ }^{1}$ MIT CSAIL, ${ }^{2}$ Qatar Computing Research Institute, HBKU \\ ${ }^{1}$ \{favyen,songtao,satvat,alizadeh,hari,madden\}@csail.mit.edu, ${ }^{2}\{$ schawla,msadeghi\}@hbku.edu.qa
}

\begin{abstract}
Accurately maintaining digital street maps is labor-intensive. To address this challenge, much work has studied automatically processing geospatial data sources such as GPS trajectories and satellite images to reduce the cost of maintaining digital maps. An end-toend map update system would first process geospatial data sources to extract insights, and second leverage those insights to update and improve the map. However, prior work largely focuses on the first step of this pipeline: these map extraction methods infer road networks from scratch given geospatial data sources (in effect creating entirely new maps), but do not address the second step of leveraging this extracted information to update the existing map data. In this paper, we first explain why current map extraction techniques yield low accuracy when extended to update existing maps. We then propose a novel method that leverages the progression of satellite imagery over time to substantially improve accuracy. Our approach first compares satellite images captured at different times to identify portions of the physical road network that have visibly changed, and then updates the existing map accordingly. We show that our change-based approach reduces error rates four-fold.
\end{abstract}

\section{CCS CONCEPTS}

- Applied computing $\rightarrow$ Cartography.

\section{KEYWORDS}

automatic map update, machine learning

\section{ACM Reference Format:}

Favyen Bastani ${ }^{1}$, Songtao $\mathrm{He}^{1}$, Satvat Jagwani ${ }^{1}$, Mohammad Alizadeh ${ }^{1}$, Hari Balakrishnan ${ }^{1}$, Sanjay Chawla ${ }^{2}$, Sam Madden ${ }^{1}$, Mohammad Amin Sadeghi ${ }^{2}$. 2021. Updating Street Maps using Changes Detected in Satellite Imagery. In 29th International Conference on Advances in Geographic Information Systems (SIGSPATIAL '21), November 2-5, 2021, Beijing, China. ACM, New York, NY, USA, 4 pages. https://doi.org/10.1145/3474717.3483651

\section{INTRODUCTION}

Maintaining street maps is labor-intensive. As a result, many techniques have been proposed to automate parts of this process by using geospatial data sources. Current map extraction techniques [1,

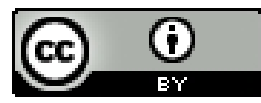

This work is licensed under a Creative Commons Attribution International 4.0 License. SIGSPATIAL '21, November 2-5, 2021, Beijing, China

(C) 2021 Copyright held by the owner/author(s).

ACM ISBN 978-1-4503-8664-7/21/11.

https://doi.org/10.1145/3474717.3483651
$3,4,10]$ primarily rely on satellite imagery due to its global availability, while some techniques use GPS trajectories.

A key problem with current techniques is that they are designed to infer road networks from scratch - however, given that we already have existing high quality maps that cover the vast majority of the world, these inferred road networks are not directly useful. Instead, an end-to-end map update system must process geospatial data sources to update and improve existing digital maps.

\subsection{Map Extraction Methods Perform Poorly on Map Update}

We first consider extending current map extraction techniques for updating maps. We will show that these methods perform poorly on map update, creating many false positive updates.

Suppose that the current live digital map has a set of roads $R$. We begin by applying a map extraction method to process the most recent satellite imagery (spanning the world). This method produces another set of roads $T$ detected in the satellite imagery. Simply replacing $R$ with $T$ would not be sensible for several reasons:

(1) For roads that appear in both $R$ and $T$, given that $R$ is largely human-curated, it captures those roads substantially more accurately than $T$.

(2) $R$ includes roads such as tunnels that cannot be detected by the map extraction method.

(3) Roads in $R$ are labeled with rich annotations such as street names, speed limits, etc. that would be lost.

We could instead try to combine $R$ and $T$ : if for a road segment $s$, $s \notin R$ and $s \in T$, we add $s$ to the map. However, in practice, this approach yields many false positives, where the map extraction method erroneously outputs road segments in places where there are no roads. We demonstrate this issue by using a state-of-the-art inference method, MAiD [2], to update OpenStreetMap [7]. We select a region of Massachusetts where OpenStreetMap has high coverage, and manually remove 204 groups of roads from the map that were constructed between 2015 and 2017. We apply MAiD to recover these removed roads, and score its performance in terms of precision and recall comparing recovered groups of roads to the manually removed groups. At $80 \%$ recall, MAiD yields only $67 \%$ precision - far too low for full automation to be a realistic option. We show examples of incorrect detections in Figure 1.

Many of these detections, such as the Runway, Walkway, and Crop Field examples in Figure 1, arise due to paths that are virtually indistinguishable from roads: it is restrictions set by policy governing the use of those paths, and not physical characteristics of the paths, that make them unsuitable for traversal by motor vehicles. Thus, simply improving the machine learning techniques 


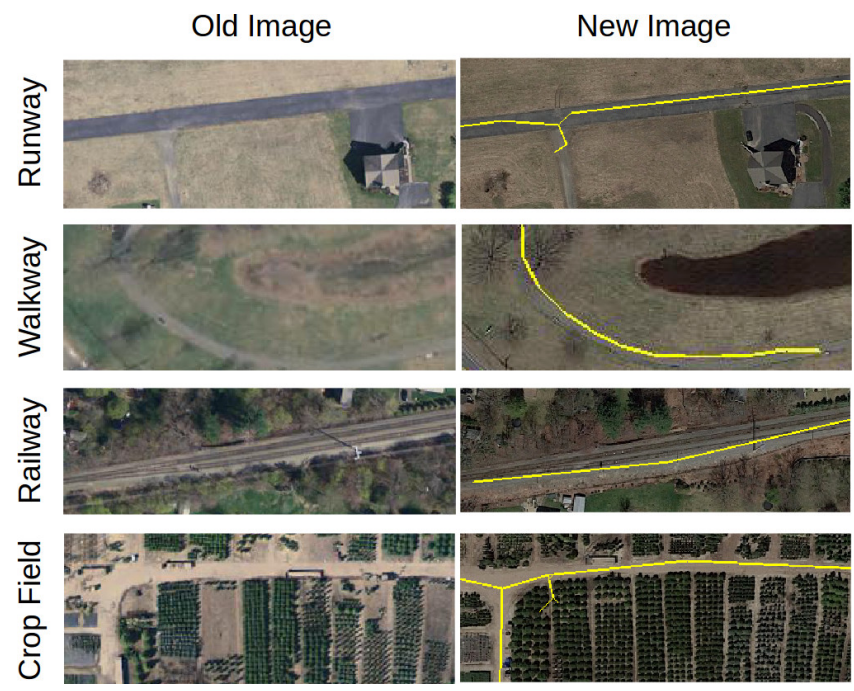

Figure 1: False positive detections when using MAiD to update OpenStreetMap. We show new 2017 imagery, in which the model erroneously detects roads, on the right. We include older 2015 imagery on the left to show how change detection could have eliminated all of these false positives by comparing satellite images over time and determining that these are not recent road network changes.

and models used in map extraction methods is unlikely to improve accuracy; instead, a fundamental shift in approach is required.

\subsection{Key Tasks in Maintaining Maps}

To determine how accuracy can be improved, we first take a step back and identify the major challenges associated with maintaining maps. In particular, digital maps have near-complete coverage in most parts of the world: a 2015 study $^{1}$ found that, in 154 of 233 considered countries and territories, the length of roads in OpenStreetMap exceeded the estimated road network length reported in the CIA World Factbook, implying that the map provided excellent coverage in those countries. Then, given that digital maps have good coverage, identifying pre-existing roads constructed several years ago is not a key issue: in almost all cases, such roads already appear in the map.

Instead, the key challenge in maintaining maps is keeping the map up-to-date with changes in the physical road network. Indeed, in the US alone, an estimated $30 \mathrm{~K} \mathrm{~km}$ of roads are constructed each year ${ }^{2}$, and map vendors spend hundreds of millions of dollars annually to keep maps up-to-date.

This presents the question: Can we develop techniques that directly tackle the key challenge of identifying physical road network changes, to substantially improve accuracy at maintaining maps over map extraction methods?

\footnotetext{
${ }^{1}$ See https://blog.mapbox.com/how-complete-is-openstreetmap-7c369787af6e.

2"Public Road Mielage", FHWA, https://www.fhwa.dot.gov/policyinformation/ statistics/2013/vmt422c.cfm.
}

\subsection{Map Update through Change Detection}

To substantially improve accuracy, we propose leveraging a source of data largely overlooked in prior work: the progression of satellite imagery over time. By comparing satellite images captured at different times, we can hone in on portions of the road network that visibly changed over the satellite image time series. Focusing on segments that visibly changed over time enables us to disambiguate false positive road segments from genuinely new constructions: for example, all of the false positive detections in Figure 1 arose from curvilinear features such as walkways and crop field paths that did not undergo any recent change; thus, by processing the satellite image time series, we can determine that these are pre-existing features, and should not be added to the map.

To implement the proposed solution, we must detect changed road segments across satellite images. However, most existing change detection methods are fully supervised. They rely on collecting annotated pairs of images where change has occurred. Since newly constructed roads are rare relative to the size of the map, collecting positive examples of new roads for such a dataset is tedious. Additionally, the diversity in visual appearance of roads makes change detection especially challenging. Furthermore, in Section 4, we show that prior work in unsupervised change detection exhibits low accuracy when applied for detecting new construction.

Instead, we develop a two-stage approach that requires no handlabeling for comparing satellite images over time to detect new roads. We evaluate our approach on a large-scale dataset consisting of $4800 \mathrm{~km}^{2}$ of satellite imagery. We apply methods to improve an existing map dataset, OpenStreetMap, by adding newly constructed roads. At $50 \%$ recall, our approach reduces error rates over existing state-of-the-art map inference methods four-fold, from $12 \%$ to $3 \%$. Our code and data is available at https://favyen.com/mapupdate.

\section{RELATED WORK}

Automatically inferring roads from satellite imagery is a wellstudied problem. Recent road extraction methods generally apply convolutional neural networks (CNNs) to segment imagery for roads, and apply various methods to post-process the segmentation output and derive vector road network data. Cheng et al. apply thresholding, thinning, and line following to extract a road network from segmentation probabilities [4]. DeepRoadMapper [10] proposes several additional refinement steps, including removing short edges and identifying potential missed roads. Some methods propose alternatives to extracting roads through segmentation. Alshehhi et al. build the road network with a region adjacent graph that forms narrow elongated regions along roads [1]. RoadTracer [3] and PolyMapper [9] propose an iterative tracing framework to extract road networks: they train a $\mathrm{CNN}$ to output the directionality of roads at each pixel, and employ an iterative search guided by the $\mathrm{CNN}$ to trace the road network. VecRoad extends the iterative tracing approach with a flexible step size and joint learning tasks [11], and Neural Turtle Graphics extends it with a sequential generative model [5]. Another recent technique, Sat2Graph, proposes a one-shot road extraction process where a $\mathrm{CNN}$ directly predicts the positions of road network vertices and edges [8].

However, broadly, these approaches are unable to reason about false positive detections made by the $\mathrm{CNN}$ such as those in Figure 


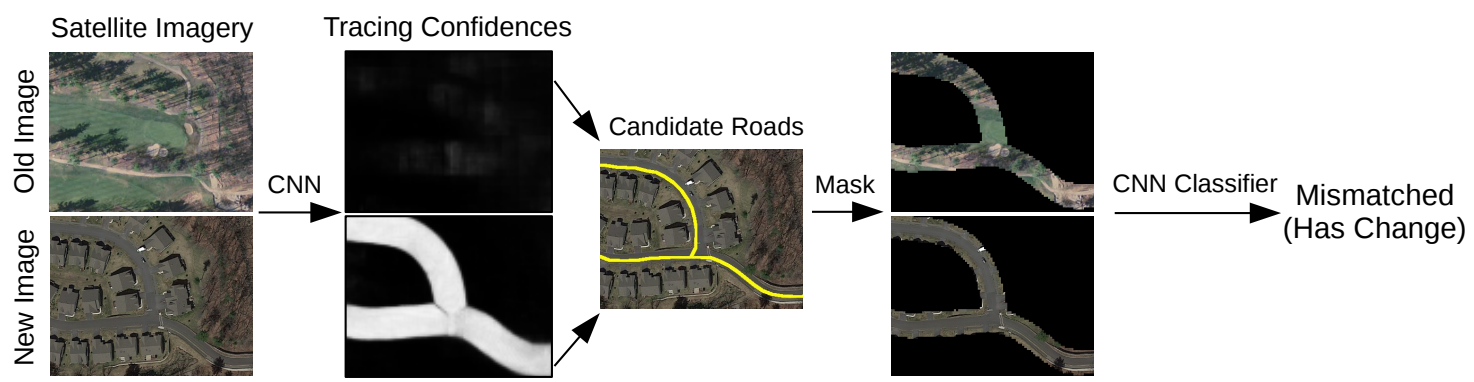

Figure 2: Our two-stage approach for detecting newly constructed roads in satellite imagery. Here, a pair of aligned images reflecting a new neighborhood is correctly detected.
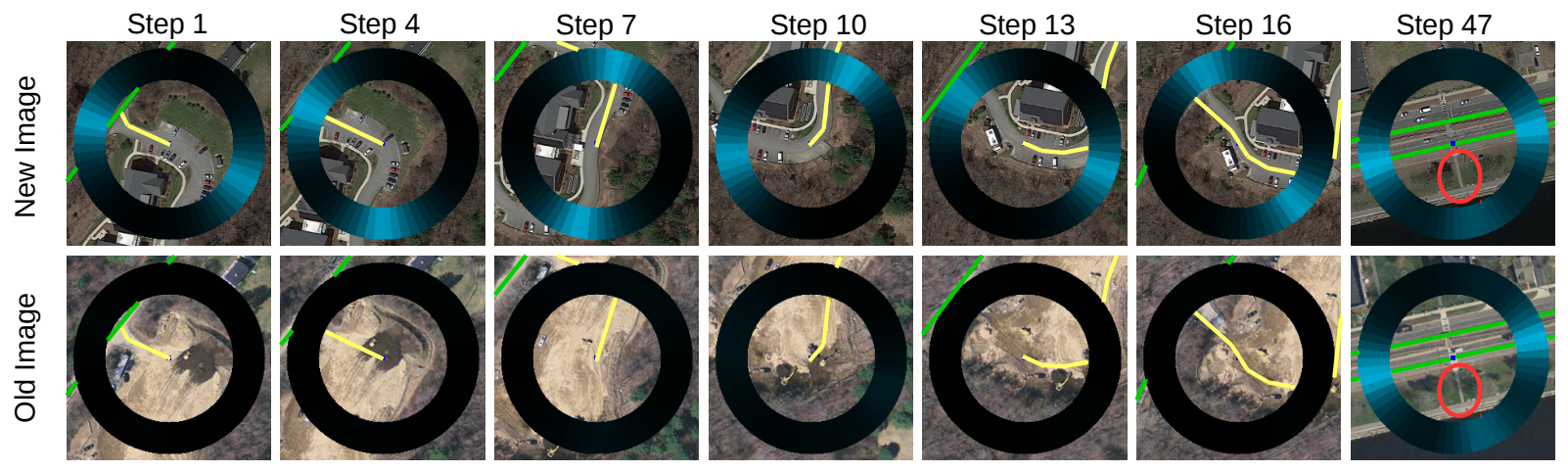

Figure 3: Illustration of change-seeking iterative tracing. The blue circle visualizes tracing confidences at the center pixel, with lighter colors indicating higher confidence. The existing map is green, and traced roads are yellow. On each step, we compare confidences in $M_{\text {new }}$ (top) against confidences in $M_{\text {old }}$ (bottom). On the right, this comparison helps to avoid tracing a pre-existing pedestrian path (red circle) when the model outputs similar confidences in both images.

1, especially for paths that appear visually similar to roads in the satellite image but are not suitable for motor vehicles. As a result, when road extraction methods are applied for the practical task of updating existing maps, they incorporate many non-road paths into the map, thereby deteriorating the quality of the map data. In contrast, in most of the world where existing maps have good coverage, our method accurately keeps maps up to date with new construction by comparing satellite imagery over time, and only updating the map in areas where change is detected across images.

\section{DETECTING STREET NETWORK CHANGES}

We propose a novel approach that directly detects newly constructed roads by comparing an up-to-date satellite image against an old image. Figure 2 summarizes our approach.

Let $M_{\text {old }}$ and $M_{\text {new }}$ be old and up-to-date satellite images of the same region, and let $G$ be the road network graph of that region in the existing map. Each vertex $v \in G$ is annotated with a pixel $(i, j)$ corresponding to its location in the images, and edges correspond to roads. At a high level, in our approach, we detect new roads by identifying roads that appear in $M_{\text {new }}$ but not in $M_{\text {old }}$ or $G$. In most of the world where existing maps have good coverage, roads detected in both $M_{\text {new }}$ and $M_{\text {old }}$ likely are not roads at all, but instead non-road paths (e.g., the examples in Figure 1); by comparing $M_{\text {new }}$ and $M_{\text {old }}$, our approach avoids these false positives.

We first apply a novel change-seeking iterative tracing procedure that adapts MAiD [2] to selectively trace roads in $M_{\text {new }}$ that appear in neither $M_{\text {old }}$ nor the existing map $G$, i.e., roads that were constructed after $M_{\text {old }}$ was captured. At a high level, we train a CNN model to predict the directionality of roads incident at each pixel in the satellite image, and then extend the existing map by tracing new roads along directions where the model has high confidence in $M_{\text {new }}$ and low confidence in $M_{\text {old }}$. Figure 3 summarizes the tracing process. Although this procedure improves precision over prior work, we find that it produces false positives when differences in off-nadir angle and lighting or visible non-construction activity result in a sharp increase in the $\mathrm{CNN}$ confidence from $M_{\text {old }}$ to $M_{\text {new }}$ despite no new roads.

Thus, we propose a novel self-supervised change detection method to automatically prune these remaining false positives in the second stage of our approach. Our method selectively identifies road network changes so that visible non-construction activity does not result in a false positive. At a high level, we train a classifier that inputs a pair of windows of old and up-to-date images. The input may either be a matching pair, where $M_{\mathrm{old}}$ and $M_{\text {new }}$ are cropped at the same window, or a mismatched pair, where $M_{\text {old }}$ and $M_{\text {new }}$ are cropped at disjoint windows. We train the classifier 

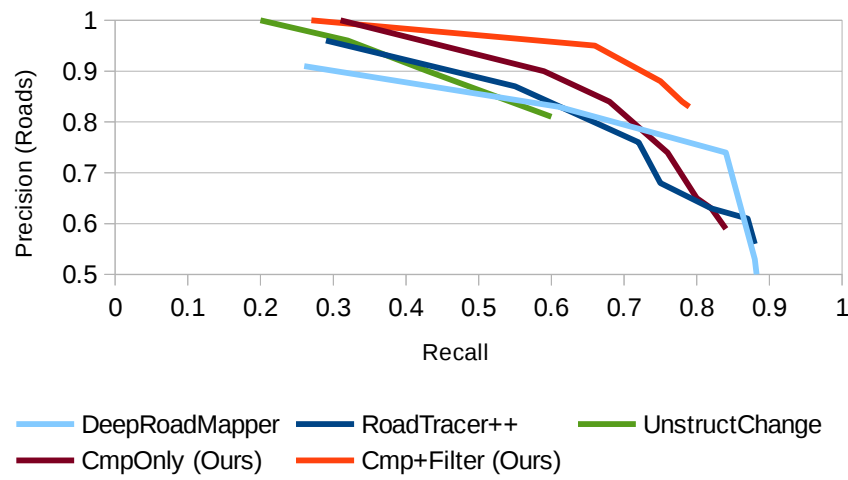

Figure 4: Precision and recall comparing detections against hand-labeled new construction.

to distinguish matching pairs from mismatched pairs. We generate training examples by deciding to create a matching pair or mismatched pair with equal probability. The classifier learns to match features between the images to determine whether they are taken at the same window despite differences in matching pairs such as shadows, camera angle, and non-construction activity that make unsupervised change detection methods ineffective on this task.

The final road detections remaining after both stages have high precision, and can be used to improve real-world maps through automatic merging or human validation.

\section{EVALUATION}

We evaluate our approach against existing state-of-the-art map inference and change detection methods on a task involving automatically updating OpenStreetMap with new roads. We use 60 $\mathrm{cm} /$ pixel resolution satellite imagery from MassGIS from 2015 and 2017 as our old imagery $M_{\text {old }}$ and up-to-date imagery $M_{\text {new }}$, and the OpenStreetMap dataset as our road network graph. We select two disjoint sections of this dataset for training $\left(3000 \mathrm{~km}^{2}\right.$ in the Boston metro area) and for evaluation $\left(1800 \mathrm{~km}^{2}\right.$ in northeastern Massachusetts).

Metrics. For evaluation, we hand-annotated 204 groups of roads that appear in $M_{\text {new }}$ but not $M_{\text {old }}$. We prune these roads from OpenStreetMap to derive a road network $G$ corresponding to a map that has not yet been updated with the new imagery. We compare the methods in terms of the precision and recall on recovering the pruned roads.

Baselines. We evaluate our method against two baselines detailed in Section 2 that implement existing state-of-the-art road inference approaches: MAiD [2] and DeepRoadMapper [10]. We apply these methods on $M_{\text {new }}$ to derive proposed roads, and prune proposals that correspond to roads already mapped in OpenStreetMap. We also evaluate against an unsupervised satellite image change detection method, UnstructChange [6], which identifies change by comparing feature maps extracted from old and up-to-date satellite images through a VGG-19 model trained for segmentation. Finally, a fourth baseline, denoted CmpOnly, applies the first stage of our approach only (change-seeking iterative tracing). We denote our full approach Cmp+Filter.
Results. We show precision-recall curves on detecting new roads over varying confidence thresholds in Figure 4. DeepRoadMapper is unable to achieve higher than $91 \%$ precision due to false positives, many of which correspond to pre-existing non-road paths. MAiD provides higher precision at lower recalls, but still yields only $88 \%$ precision at $50 \%$ recall. At $50 \%$ recall, CmpOnly improves precision to $94 \%$, and $\mathrm{Cmp}+$ Filter further improves precision to $97 \%$. Thus, our method effectively prunes false positives that have lighting and angle differences or visible activity but no new roads. UnstructChange does not improve performance over MAiD: it outputs false positives due to non-construction changes (such as angle and lighting differences) between the old and up-to-date images.

\section{CONCLUSION}

Maintaining street maps today is labor-intensive and costly. We find that existing state-of-the-art street map inference systems exhibit low precision when applied to update an existing map dataset, OpenStreetMap. Precision is crucial because automatic integration of detections into the street map dataset is only practical if errors are rare - otherwise, the confusion for users caused by introducing errors may outweigh the benefit from expanded map coverage. By leveraging multiple satellite images collected at different times, our two-stage approach complements prior work by identifying roads and buildings that were newly constructed in the most recent image. Our evaluation on $4800 \mathrm{~km}^{2}$ of satellite imagery shows that our approach is able to update existing maps to capture new construction with high precision.

\section{REFERENCES}

[1] Rasha Alshehhi, Prashanth Reddy Marpu, Wei Lee Woon, and Mauro Dalla Mura. 2017. Simultaneous Extraction of Roads and Buildings in Remote Sensing Imagery with Convolutional Neural Networks. ISPRS fournal of Photogrammetry and Remote Sensing 130 (2017), 139-149.

[2] Favyen Bastani, Songtao He, Sofiane Abbar, Mohammad Alizadeh, Hari Balakrishnan, Sanjay Chawla, and Sam Madden. 2018. Machine-Assisted Map Editing. In ACM International Conference on Advances in Geographic Information Systems (SIGSPATIAL). 23-32.

[3] Favyen Bastani, Songtao He, Sofiane Abbar, Mohammad Alizadeh, Hari Balakrishnan, Sanjay Chawla, Sam Madden, and David DeWitt. 2018. RoadTracer: Automatic Extraction of Road Networks from Aerial Images. In CVPR

[4] Guangliang Cheng, Ying Wang, Shibiao Xu, Hongzhen Wang, Shiming Xiang, and Chunhong Pan. 2017. Automatic Road Detection and Centerline Extraction via Cascaded End-to-End Convolutional Neural Network. IEEE Transactions on Geoscience and Remote Sensing 55, 6 (2017), 3322-3337.

[5] Hang Chu, Daiqing Li, David Acuna, Amlan Kar, Maria Shugrina, Xinkai Wei, Ming-Yu Liu, Antonio Torralba, and Sanja Fidler. 2019. Neural Turtle Graphics for Modeling City Road Layouts. In Proceedings of the IEEE International Conference on Computer Vision (ICCV). 4522-4530.

[6] Kevin Louis de Jong and Anna Sergeevna Bosman. 2019. Unsupervised Change Detection in Satellite Images Using Convolutional Neural Networks. In International foint Conference on Neural Networks (IFCNN). IEEE, 1-8.

[7] Mordechai Haklay and Patrick Weber. 2008. OpenStreetMap: User-Generated Street Maps. IEEE Pervasive Computing 7, 4 (2008), 12-18.

[8] Songtao He, Favyen Bastani, Satvat Jagwani, Mohammad Alizadeh, Hari Balakrishnan, Sanjay Chawla, Mohamed M Elshrif, Samuel Madden, and Amin Sadeghi. 2020. Sat2Graph: Road Graph Extraction through Graph-Tensor Encoding. Proceedings of the European Conference on Computer Vision (ECCV) (2020).

[9] Zuoyue Li, Jan Dirk Wegner, and Aurélien Lucchi. 2019. Topological Map Extraction from Overhead Images. In Proceedings of the IEEE International Conference on Computer Vision (ICCV). 1715-1724.

[10] Gellért Máttyus, Wenjie Luo, and Raquel Urtasun. 2017. DeepRoadMapper: Extracting Road Topology From Aerial Images. In CVPR

[11] Yong-Qiang Tan, Shang-Hua Gao, Xuan-Yi Li, Ming-Ming Cheng, and Bo Ren. 2020. VecRoad: Point-based Iterative Graph Exploration for Road Graphs Extraction. In Proceedings of the IEEE Conference on Computer Vision and Pattern Recognition (CVPR). 8910-8918. 